\title{
Membangun Fungsi Green dari Persamaan Difrensial Linear Non Homogen Tingkat - $n$
}

\author{
Eddy Djauhari \\ Program Studi S1 Matematika FMIPA Universitas Padjadjaran \\ Jalan Raya Bandung Sumedang KM.21, Jatinangor Sumedang 45363 \\ Email : eddymath2014@gmail.com
}

\begin{abstract}
ABSTRAK
Dalam makalah ini akan disajikan bagaimana membangun fungsi Green dari persamaan diferensial linear non homogen tingkat- $n$. Salah satu metodenya adalah melalui metode variasi parameter. Solusi umum dari persamaan diferensial linear non homogen tingkat-n terdiri dari solusi homogen dan solusi non homogen. Solusi non homogen sering juga disebut solusi partikulir. Selanjutnya dari solusi partikulir inilah dapat dibangun fungsi Green yang memenuhi beberapa syarat. Fungsi Green ini selain dapat digunakan untuk mencari solusi dari persamaan diferensial linear nonhomogen tingkat- $n$, juga banyak digunakan dalam bidang fisika, elektro, komputer dan sebagainya. Dalam makalah ini pula akan diberikan contoh mencari penyelesaian umum dari persamaan diferensial linear non homogen tingkat- $n$ dengan fungsi Green.
\end{abstract}

Kata kunci: persamaan diferensial, solusi homogen, Wronsky, solusi khusus, variasi parameter dan fungsi Green.

\section{ABSTRACT}

In this paper, we will be presented how to construct the Green function from nonhomogeneous nth-onder linear differential equation. One of the methods is by using the method of variation of parameters. The general solution of nonhomogeneous nth-order linear differential equation consist of homogeneous solution and nonhomogeneous solution. Nonhomogeneous solution is also called particular solution. Next, we can construct the Green function from the particular solution, where the Green function must hold some conditions. The Green function beside can be used for finding the solution of nonhomogeneous nth-order linear differential equation, also can be used in physics, electrical, computer, etc. In this paper, we give also some examples for finding the general solution of nonhomogeneous nth-order linear differential equation by the Green function.

Keywords: differential equation, homogeneous solution, Wronsky, particular solution, variation of parameter, and Green function.

\section{Pendahuluan}

Pandang persamaan diferensial linear non homogen tingkat- $n$ :

$$
y^{(n)}+a_{1}(x) y^{(n-1)}+a_{2}(x) y^{(n-2)}+\cdots+a_{n}(x) y=f(x)
$$

dengan fungsi $f$ kontinu pada daerah definisinya. Fungsi Green untuk persamaan diferensial di atas dapat dicari sehingga dengan mudah menentukan solusi umum persamaan diferensial untuk fungsi $f$ sembarang. Dalam makalah ini akan diperkenalkan membangun fungsi Green dari persamaan diferensial linear melalui metode variansi parameter/konstanta.

\section{Konsep Fungsi Green}

Pandang persamaan diferensial linear non homogen tingkat- $n$ :

$$
y^{n}+a_{1}(x) y^{(n-1)}+a_{2}(x) y^{n-2}+\cdots+a_{n}(x) y=f(x)
$$

Fungsi $G(x, t)$ dikatakan fungsi Green untuk masalah nilai awal persamaan diferensial di atas jika memenuhi kondisi sebagai berikut :

1. $G(x, t)$ terdefinisi pada daerah $\mathbb{R}=I$ untuk $x \in I$ dari semua titik $(x, t)$ dengan $x$ dan $t$ terletak dalam selang I.

2. $G(x, t), \frac{\partial G}{\partial x}, \frac{\partial^{2} G}{\partial x^{2}}, \ldots, \frac{\partial^{n} G}{\partial x^{n}}$ merupakan fungsi yang kontinu pada $\mathbb{R}=\mathrm{I} \times \mathrm{I}$

3. Untuk setiap $x_{0}$ dalam selang $I$ dan fungsi $f \in C(\mathrm{I})$, fungsi 


$$
y_{p}(x)=\int_{x_{0}}^{x} G(x, t) f(t) d t
$$

adalah solusi persamaan diferensial (1) yang memenuhi kondisi awal $y_{p}\left(x_{0}\right)=y_{p}^{\prime}\left(x_{0}\right)=$ $y_{p}^{\prime \prime}\left(x_{0}\right)=\cdots=y_{p}^{(n-1)}\left(x_{0}\right)=0[2]$.

\section{Membangun Fungsi Green Dari Persamaan Diferensial Linear Non Homogen Tingkat-n}

Pandang persamaan diferensial linear non homogen tingkat- $n$ :

$$
y^{(n)}+a_{1}(x) y^{(n-1)}+a_{2}(x) y^{(n-2)}+\cdots+a_{n}(x) y=f(x)
$$

Solusi umum persamaan diferensial di atas adalah $y=y_{h}+y_{p}$, dengan $y_{h}$ merupakan solusi umum persamaan diferensial homogen dan $y_{p}$ adalah solusi khusus atau solusi partikulir. Misalkan $y_{1}(x), y_{2}(x), \ldots, y_{n}(x)$ solusi basis untuk persamaan diferensial homogennya, maka

$$
y_{h}=c_{1} y_{1}(x)+c_{2} y_{2}(x)+\cdots+c_{n} y_{n}(x)
$$

dengan $c_{1}, c_{2}, \ldots, c_{n}$ merupakan bilangan konstanta. Misalkan solusi khususnya:

$$
y_{p}=u_{1}(x) y_{1}+u_{2}(x) y_{2}+\cdots+u_{n}(x) y_{n}
$$

dengan $u_{1}^{\prime}, u_{2}^{\prime}, \ldots, u_{n}^{\prime}$ ditentukan dari sistem persamaan yang terdiri dari $n$ persamaan :

$$
\left\{\begin{array}{cccc}
u_{1}^{\prime} y_{1}+u_{2}^{\prime} y_{2}+\cdots+u_{n}^{\prime} y_{n} & =0 \\
u_{1}^{\prime} y_{1}^{\prime}+u_{2}^{\prime} y_{2}^{\prime}+\cdots+u_{n}^{\prime} y_{n}^{\prime} & =0 \\
\vdots & \vdots & \vdots & \vdots \\
u_{1}^{\prime} y_{1}^{(n-1)}+u_{2}^{\prime} y_{2}^{(n-1)}+\cdots+u_{n}^{\prime} y_{n}^{(n-1)} & =f(x)
\end{array}\right.
$$

Dengan menggunakan aturan Cramer, maka diperoleh:

$u_{k}^{\prime}=\frac{\left|\begin{array}{cccccccc}y_{1} & y_{2} & \ldots & y_{k-1} & 0 & y_{k+1} & \ldots & y_{n} \\ y_{1}^{\prime} & y_{2}^{\prime} & \ldots & y_{k-1}^{\prime} & 0 & y_{k+1}^{\prime} & \ldots & y_{n}^{\prime} \\ \vdots & \vdots & & \vdots & \vdots & \vdots & & \vdots \\ y_{1}^{(n-1)} & y_{1}^{(n-1)} & \ldots & y_{k-1}^{(n-1)} & f & y_{k+1}^{(n-1)} & \ldots & y_{1}^{(n-1)}\end{array}\right|}{\begin{array}{ccccc}y_{1} & y_{2} & \ldots & y_{n} & \\ y_{1}^{\prime} & y_{2}^{\prime} & \ldots & y_{n}^{\prime} \\ \vdots & \vdots & & \vdots \\ y_{1}^{(n-1)} & y_{1}^{(n-1)} & \ldots & y_{1}^{(n-1)}\end{array} \mid}$

dengan $k=1,2, \ldots, n$

Misalkan $W\left[y_{1}(x), y_{2}(x), \ldots, y_{n}(x)\right]$ merupakan determinan Wronsky dengan

$$
W\left[y_{1}(x), y_{2}(x), \ldots y_{n}(x)\right]=\left|\begin{array}{cccc}
y_{1} & y_{2} & \ldots & y_{n} \\
y_{1}^{\prime} & y_{2}^{\prime} & \ldots & y_{n}^{\prime} \\
\vdots & \vdots & & \vdots \\
y_{1}^{(n-1)} & y_{2}^{(n-1)} & \ldots & y_{n}^{(n-1)}
\end{array}\right|
$$

Misalkan pula $\mathrm{V}_{\mathrm{k}}(\mathrm{x})$ merupakan determinan yang diperoleh dari $W\left[y_{1}(x), y_{2}(x), \ldots . . y_{n}(x)\right]$ dengan menggantikan kolom, ke $-k$ dengan $\left[\begin{array}{c}0 \\ 0 \\ \vdots \\ 1\end{array}\right]$ Jadi $V_{k}(x)=\left|\begin{array}{cccccccc}y_{1} & y_{2} & \ldots & y_{k-1} & 0 & y_{k+1} & \ldots & y_{n} \\ y_{1}^{\prime} & y_{2}^{\prime} & \ldots & y_{k-1}^{\prime} & 0 & y_{k+1}^{\prime} & \ldots & y_{n}^{\prime} \\ \vdots & \vdots & & \vdots & \vdots & \vdots & & \vdots \\ y_{1}^{(n-1)} & y_{1}^{(n-1)} & \ldots & y_{k-1}^{(n-1)} & 1 & y_{k+1}^{(n-1)} & \ldots & y_{n}^{(n-1)}\end{array}\right|$ dengan $k=1,2, \ldots, n$

Jadi persamaan (4) dapat ditulis menjadi 


$$
\begin{aligned}
u_{k}^{\prime} & =\frac{\left|\begin{array}{cccccccc}
y_{1} & y_{2} & \ldots & y_{k-1} & 0 & y_{k+1} & \ldots & y_{n} \\
y_{1}^{\prime} & y_{2}^{\prime} & \ldots & y_{k-1}^{\prime} & 0 & y_{k+1}^{\prime} & \ldots & y_{n}^{\prime} \\
\vdots & \vdots & & \vdots & \vdots & \vdots & & \vdots \\
y_{1}^{(n-1)} & y_{1}^{(n-1)} & \ldots & y_{k-1}^{(n-1)} & 1 & y_{k+1}^{(n-1)} & \ldots & y_{n}^{(n-1)}
\end{array}\right| \cdot f(x)}{\mathrm{W}\left[y_{1}(x), y_{2}(x), \ldots, y_{n}(x)\right]} \\
u_{k}^{\prime} & =\frac{V_{k}(x) \cdot f(x)}{\mathrm{W}\left[y_{1}(x), y_{2}(x), \ldots, y_{n}(x)\right]}
\end{aligned}
$$

Jadi diperoleh

$$
u_{k}=\int_{x_{0}}^{x} \frac{V_{k}(t) \cdot f(t)}{\mathrm{W}\left[y_{1}(t), y_{2}(t), \ldots, y_{n}(t)\right]} d t, \quad \text { dengan } k=1,2, \ldots, n
$$

Mensubsitusikan (5) ke dalam (3) diperoleh :

dimana

$$
\begin{gathered}
y_{p}=\int_{x_{0}}^{x} \frac{y_{1}(x) V_{1}(t)+\cdots+y_{n}(x) V_{n}(t)}{W\left[y_{1}(t), y_{2}(t), \ldots, y_{n}(t)\right]} \cdot f(t) d t \\
y_{p}=\int_{x_{0}}^{\mathrm{x}} G(x, t) \cdot f(t) d t
\end{gathered}
$$

$$
G(x, t)=\frac{y_{1}(x) V_{1}(t)+\cdots+y_{n}(x) V_{n}(t)}{W\left[y_{1}(t), y_{2}(t), \ldots, y_{n}(t)\right]}
$$

Jadi solusi umum persamaan diferensial (2) adalah

$$
y=y_{h}+\int_{x_{0}}^{x} G(x, t) \cdot f(t) d t \quad[3]
$$

Akan ditunjukkan bahwa $G(x, t)$ yang didefinisikan oleh (6) merupakan fungsi Green untuk persamaan diferensial (2). Jelas bahwa

$$
\int_{x_{0}}^{x} G(x, t) \cdot f(t) d t
$$

merupakan solusi khusus dari persamaan diferensial (2) dan $\mathrm{G}(\mathrm{x}, \mathrm{t})$ memenuhi hal-hal berikut :

1. $G(x, t)$ terdefinisi untuk setiap $(x, t)$ karena $W\left[y_{1}(t), y_{2}(t), \ldots, y_{n}(t)\right] \neq 0$, untuk setiap $t \in$ $\left[x_{0}, x\right]$.

2. $G(x, t)$ kontinu untuk setiap $(x, t)$, karena $V_{1}(t), V_{2}(t), \ldots, V_{n}(t), y_{1}(x), y_{2}(x), \ldots, y_{n}(x)$ kontinu untuk setiap $(x, t) . V_{1}(t), V_{2}(t), \ldots, V_{n}(t)$ kontinu, karena $y_{1}, y_{2}, \ldots, y_{n}$ dan turunan-turunannya kontinu sampai dengan tingkat ke $n-1$.

$\frac{\partial G}{\partial x}$ kontinu untuk setiap $(x, t)$, karena $V_{1}(t), V_{2}(t), \ldots, V_{n}(t), y_{1}^{\prime}(x), y_{2}^{\prime}(x), \ldots, y_{n}^{\prime}(x)$ kontinu untuk $\operatorname{setiap}(x, t)$.

$\frac{\partial^{2} G}{\partial x^{2}}$ kontinu untuk setiap $(\mathrm{x}, \mathrm{t})$, karena $V_{1}(t), V_{2}(t), \ldots, V_{n}(t), y_{1}^{\prime \prime}(x), y_{2}^{\prime \prime}(x), \ldots, y_{n}^{\prime \prime}(x)$ kontinu untuk setiap $(x, t)$.

$$
\frac{\partial^{n} G}{\partial x^{n}} \text { kontinu untuk setiap }(x, t) \text {, karena } V_{1}(t), V_{2}(t), \ldots, V_{n}(t), y_{1}^{(n)}(x), y_{2}^{(n)}(x), \ldots, y_{n}^{(n)}(x) \text { kontinu }
$$
untuk setiap $(x, t)$.

3. Dari bangun $y_{p}$, terlihat bahwa

$$
y_{p}(x)=\int_{x_{0}}^{x} G(x, t) \cdot f(t) d t
$$

adalah solusi khusus dari persamaan diferensial (2). Jelas bahwa

$$
y_{p}\left(x_{0}\right)=\int_{x_{0}}^{x_{0}} G(x, t) \cdot f(t) d t=0
$$




$$
y_{p}^{\prime}(x)=\int_{x_{0}}^{x} \frac{\partial G}{\partial x} f(t) d t+G(x, x) \cdot f(x)
$$

Akan dibuktikan $G(x, x)=0$, untuk setiap $x$

$$
G(x, x)=\frac{y_{1}(x) V_{1}(x)+y_{2}(x) V_{2}(x)+\cdots+y_{n}(x) V_{n}(x)}{W\left[y_{1}(x), y_{2}(x), \ldots, y_{n}(x)\right]}=0
$$

$y_{1} V_{1}+y_{2} V_{2}+\cdots+y_{n} V_{n}$

$$
=y_{1}\left|\begin{array}{cccc}
0 & y_{2} & \cdots & y_{n} \\
0 & y_{2}^{\prime} & \cdots & y_{n}^{\prime} \\
\vdots & \vdots & & \vdots \\
1 & y_{2}^{(n-1)} & \cdots & y_{n}^{(n-1)}
\end{array}\right|+y_{2}\left|\begin{array}{cccc}
y_{1} & 0 & \cdots & y_{n} \\
y_{1}^{\prime} & 0 & \cdots & y_{n}^{\prime} \\
\vdots & \vdots & & \vdots \\
y_{1}^{(n-1)} & 1 & \cdots & y_{n}^{(n-1)}
\end{array}\right|+\cdots+y_{n}\left|\begin{array}{cccc}
y_{1} & y_{2} & \cdots & 0 \\
y_{1}^{\prime} & y_{2}^{\prime} & \cdots & 0 \\
\vdots & \vdots & & \vdots \\
y_{1}^{(n-1)} & y_{2}^{(n-1)} & \cdots & 1
\end{array}\right|
$$

Untuk $n$ ganjil maka

$y_{1} V_{1}+y_{2} V_{2}+\cdots+y_{n} V_{n}$

$=y_{1}\left|\begin{array}{cccc}y_{2} & y_{3} & \cdots & y_{n} \\ y_{2}^{\prime} & y_{3}^{\prime} & \cdots & y_{n}^{\prime} \\ \vdots & \vdots & & \vdots \\ y_{2}^{(n-2)} & y_{3}^{(n-2)} & \cdots & y_{n}^{(n-2)}\end{array}\right|-y_{2}\left|\begin{array}{cccc}y_{1} & y_{3} & \cdots & y_{n} \\ y_{1}^{\prime} & y_{3}^{\prime} & \cdots & y_{n}^{\prime} \\ \vdots & \vdots & & \vdots \\ y_{1}^{(n-2)} & y_{3}^{(n-2)} & \cdots & y_{n}^{(n-2)}\end{array}\right|+\cdots+y_{n}\left|\begin{array}{cccc}y_{1} & y_{2}^{\prime} & \cdots & y_{n-1} \\ y_{1}^{\prime} & y_{2}^{\prime} & \cdots & y_{n-1}^{\prime} \\ \vdots & \vdots & & \vdots \\ y_{1}^{(n-2)} & y_{2}^{(n-2)} & \cdots & y_{n-1}^{(n-2)}\end{array}\right|$

$=\left|\begin{array}{cccc}y_{1} & y_{2} & \cdots & y_{n} \\ y_{1} & y_{2} & \cdots & y_{n} \\ y_{1}^{\prime} & y_{2}^{\prime} & \cdots & y_{n}^{\prime} \\ \vdots & \vdots & & \vdots \\ y_{1}^{(n-2)} & y_{2}^{(n-2)} & \cdots & y_{n}^{(n-2)}\end{array}\right|$

Menurut sifat determinan, karena ada dua baris yang terdiri dari elemen-elemen yang sama, maka $y_{1} V_{1}+y_{2} V_{2}+\cdots+y_{n} V_{n}=0[4]$.

Untuk $n$ genap maka

$$
\begin{aligned}
& y_{1} V_{1}+y_{2} V_{2}+\cdots+y_{n} V_{n} \\
= & -y_{1}\left|\begin{array}{cccc}
y_{2} & y_{3} & \cdots & y_{n} \\
y_{2}^{\prime} & y_{3}^{\prime} & \cdots & y_{n}^{\prime} \\
\vdots & \vdots & & \vdots \\
y_{2}^{(n-2)} & y_{3}^{(n-2)} & \cdots & y_{n}^{(n-2)}
\end{array}\right|+y_{2}\left|\begin{array}{cccc}
y_{1} & y_{3} & \cdots & y_{n} \\
y_{1}^{\prime} & y_{3}^{\prime} & \cdots & y_{n}^{\prime} \\
\vdots & \vdots & & \vdots \\
y_{1}^{(n-2)} & y_{3}^{(n-2)} & \cdots & y_{n}^{(n-2)}
\end{array}\right|-\cdots-y_{n}\left|\begin{array}{cccc}
y_{1} & y_{2} & \cdots & y_{n-1} \\
y_{1}^{\prime} & y_{2}^{\prime} & \cdots & y_{n-1}^{\prime} \\
\vdots & \vdots & & \vdots \\
y_{1}^{(n-2)} & y_{2}^{(n-2)} & \cdots & y_{n-1}^{(n-2)}
\end{array}\right| \\
= & -\left|\begin{array}{cccc}
y_{1} & y_{2} & \cdots & y_{n} \\
y_{1} & y_{2} & \cdots & y_{n} \\
y_{1}^{\prime} & y_{2}^{\prime} & \cdots & y_{n}^{\prime} \\
\vdots & \vdots & & \vdots \\
y_{1}^{(n-2)} & y_{2}^{(n-2)} & \cdots & y_{n}^{(n-2)}
\end{array}\right|
\end{aligned}
$$

Menurut sifat determinan, karena ada dua baris yang terdiri dari elemen-elemen yang sama, maka $y_{1} V_{1}+y_{2} V_{2}+\cdots+y_{n} V_{n}=0$ [4]. Karena $W\left[y_{1}(t), y_{2}(t), \ldots, y_{n}(t)\right] \neq 0$, maka $G(x, x)=0$. Akibatnya persamaan (7) menjadi :

$$
\begin{aligned}
& y_{p}^{\prime}(x)=\int_{x_{0}}^{x} \frac{\partial G}{\partial x} f(t) d t \\
& y_{p}^{\prime}\left(x_{0}\right)=\int_{x_{0}}^{x_{0}} \frac{\partial G}{\partial x} f(t) d t=0 \\
& y_{p}^{\prime \prime}(x)=\int_{x_{0}}^{x} \frac{\partial^{2} G}{\partial x^{2}} f(t) d t+\frac{\partial G}{\partial x}(x, x) f(x)
\end{aligned}
$$




$$
\begin{aligned}
& y_{p}^{\prime \prime}\left(x_{0}\right)=\int_{x_{0}}^{x_{0}} \frac{\partial^{2} G}{\partial x^{2}} f(t) d t+0=0 \\
& \vdots \\
& y_{p}^{(n-1)}(x)=\int_{x_{0}}^{x} \frac{\partial^{n-1} G}{\partial x^{n-1}} f(t) d t+\frac{\partial^{n-2} G}{\partial x^{n-2}}(x, x) f(t) \\
& y_{p}^{(n-1)}\left(x_{0}\right)=\int_{x_{0}}^{x_{0}} \frac{\partial^{n-1} G}{\partial x^{n-1}} f(t) d t+0=0[5]
\end{aligned}
$$

Contoh 1.

Bangunlah fungsi Green dari persamaan diferensial $3 y^{\prime \prime \prime}+5 y^{\prime \prime}-2 y^{\prime}=r(x)$, tentukanlah solusi umumnya.

Persamaan diferensial homogen: $3 y^{\prime \prime}+5 y^{\prime \prime}-2 y^{\prime}=0$

Persamaan karakteristik:

$$
\begin{gathered}
3 m^{3}+5 m^{2}-2 m=0 \\
m\left(3 m^{2}+5 m-2\right)=0 \\
m(3 m-1)(m+2)=0
\end{gathered}
$$

Akar-akar karakteristik:

$$
m_{1}=0, m_{2}=-2, m_{3}=\frac{1}{3}
$$

Solusi homogen: $y_{h}=c_{1}+c_{2} e^{-2 x}+c_{3} e^{\frac{1}{3} x}$

$$
\text { dengan }\left\{\begin{array}{ccc}
y_{1}(x)=1 & y_{2}(x)=e^{-2 x} & y_{3}(x)=e^{\frac{1}{3} x} \\
y_{1}^{\prime}(x)=0 & y_{2}^{\prime}(x)=-2 e^{-2 x} & y_{3}^{\prime}(x)=\frac{1}{3} e^{\frac{1}{3} x} \\
y_{1}^{\prime \prime}(x)=0 & y_{2}^{\prime \prime}(x)=4 e^{-2 x} & y_{3}^{\prime \prime}(x)=\frac{1}{9} e^{\frac{1}{3} x}
\end{array}\right.
$$

$W\left[y_{1}(t), y_{2}(t) y_{3}(t)\right]=\left|\begin{array}{ccc}1 & e^{-2 t} & e^{\frac{1}{3} t} \\ 0 & -2 e^{-2 t} & \frac{1}{3} e^{\frac{1}{3} t} \\ 0 & 4 e^{-2 t} & \frac{1}{9} e^{\frac{1}{3} t}\end{array}\right|=\left|\begin{array}{cc}-2 e^{-2 t} & \frac{1}{3} e^{\frac{1}{3} t} \\ 4 e^{-2 t} & \frac{1}{9} e^{\frac{1}{3} t}\end{array}\right|=-\frac{14}{9} e^{-\frac{5}{3} t}$

$V_{1}(t)=\left|\begin{array}{ccc}0 & e^{-2 t} & e^{\frac{1}{3} t} \\ 0 & -2 e^{-2 t} & \frac{1}{3} e^{\frac{1}{3} t} \\ 1 & 4 e^{-2 t} & \frac{1}{9} e^{\frac{1}{3} t}\end{array}\right|=\left|\begin{array}{cc}e^{-2 t} & e^{\frac{1}{3} t} \\ -2 e^{-2 t} & \frac{1}{3} e^{\frac{1}{3} t}\end{array}\right|=\frac{7}{3} e^{-\frac{5}{3} t}$

$V_{2}(t)=\left|\begin{array}{lll}1 & 0 & e^{e^{\frac{1}{3}} t} \\ 0 & 0 & \frac{1}{3} e^{\frac{1}{3} t} \\ 0 & 1 & \frac{1}{9} e^{\frac{1}{3} t}\end{array}\right|=\left|\begin{array}{cc}0 & \frac{1}{3} e^{\frac{1}{3} t} \\ 1 & \frac{1}{9} e^{\frac{1}{3} t}\end{array}\right|=-\frac{1}{3} e^{\frac{1}{3} t}$

$V_{3}(t)=\left|\begin{array}{ccc}1 & e^{-2 t} & 0 \\ 0 & -2 e^{-2 t} & 0 \\ 0 & 4 e^{-2 t} & 1\end{array}\right|=\left|\begin{array}{cc}-2 e^{-2 t} & 0 \\ 4 e^{-2 t} & 1\end{array}\right|=-2 e^{-2 t}$

Jadi fungsi Green:

$$
\begin{aligned}
& G(x, t)=\frac{y_{1}(x) V_{1}(t)+y_{2}(x) V_{2}(t)+y_{3}(x) V_{3}(t)}{W\left[y_{1}(t), y_{2}(t), y_{3}(t)\right]} \\
& G(x, t)=\frac{1 \cdot\left(\frac{7}{3} e^{-\frac{5}{3} t}\right)+e^{-2 x}\left(-\frac{1}{3} e^{\frac{1}{3} t}\right)+e^{\frac{1}{3} x}\left(-2 e^{-2 t}\right)}{-\frac{14}{9} e^{-\frac{5}{3} t}} \\
& =-\frac{3}{2}+\frac{3}{14} e^{-2(x-t)}+\frac{9}{7} e^{\frac{1}{3}(x-t)} \\
& f(t)=\frac{r(t)}{3}
\end{aligned}
$$


Solusi khususnya:

Jadi solusi umumnya :

$$
\begin{aligned}
& y_{p}=\int_{x_{0}}^{x}\left(-\frac{3}{2}+\frac{3}{14} e^{-2(x-t)}+\frac{9}{7} e^{\frac{1}{3}(x-t)}\right) \cdot \frac{r(t)}{3} d t \\
& y_{p}=\int_{x_{0}}^{x_{x}}\left(-\frac{1}{2}+\frac{1}{14} e^{-2(x-t)}+\frac{3}{7} e^{\frac{1}{3}(x-t)}\right) \cdot r(t) d t
\end{aligned}
$$

Contoh 2.

$$
y=c_{1}+c_{2} e^{-2 x}+c_{3} e^{\frac{1}{3} x}+\int_{x_{0}}^{x}\left(-\frac{1}{2}+\frac{1}{14} e^{-2(x-t)}+\frac{3}{7} e^{\frac{1}{3}(x-t)}\right) \cdot r(t) d t
$$

Bangunlah fungsi Green dari persamaan diferensial

$$
x^{3} y^{\prime \prime \prime}+2 x^{2} y^{\prime \prime}-2 x y^{\prime}=1+\frac{1}{x}, x>0,
$$

kemudian akan ditentukan solusi umumnya.

Persamaan diferensial Cauchy homogen: $x^{3} y^{\prime \prime \prime}+2 x^{2} y^{\prime \prime}-2 x y^{\prime}=0$

Persamaan pembantunya:

$$
\begin{aligned}
& m(m-1)(m-2)+2 m(m-1)-2 m=0 \\
& m((m-1)(m-2)+2(m-1)-2)=0 \\
& m\left(m^{2}-3 m+2+2 m-2-2\right)=0 \\
& m\left(m^{2}-m-2\right)=0 \\
& m(m+1)(m-2)=0 \\
& m_{1}=0, m_{2}=-1, m_{3}=2
\end{aligned}
$$

Diperoleh solusi homogen : $y_{h}=c_{1}+c_{2} x^{-1}+c_{3} x^{2}$

$$
\begin{gathered}
\text { dengan }\left\{\begin{array}{ccc}
y_{1}(x)=1 & y_{2}(x)=x^{-1} & y_{3}(x)=x^{2} \\
y_{1}^{\prime}(x)=0 & y_{2}^{\prime}(x)=-x^{-2} & y_{3}^{\prime}(x)=2 x \\
y_{1}^{\prime \prime}(x)=0 & y_{2}^{\prime \prime}(x)=2 x^{-3} & y_{3}^{\prime \prime}(x)=2
\end{array}\right. \\
W\left[y_{1}(t), y_{2}(t), y_{3}(t)\right]=\left|\begin{array}{ccc}
1 & t^{-1} & t^{2} \\
0 & -t^{-2} & 2 t \\
0 & 2 t^{-3} & 2
\end{array}\right|=\left|\begin{array}{cc}
-t^{-2} & 2 t \\
2 t^{-3} & 2
\end{array}\right|=-6 t^{-2} \\
V_{1}(t)=\left|\begin{array}{ccc}
0 & t^{-1} & t^{2} \\
0 & -t^{-2} & 2 t \\
1 & 2 t^{-3} & 2
\end{array}\right|=\left|\begin{array}{cc}
t^{-1} & t^{2} \\
-t^{-2} & 2 t
\end{array}\right|=2+1=3 \\
V_{2}(t)=\left|\begin{array}{ccc}
1 & 0 & t^{2} \\
0 & 0 & 2 t \\
0 & 1 & 2
\end{array}\right|=\left|\begin{array}{cc}
0 & 2 t \\
1 & 2
\end{array}\right|=-2 t \\
V_{3}(t)=\left|\begin{array}{ccc}
1 & t^{-1} & 0 \\
0 & -t^{-2} & 0 \\
0 & 2 t^{-3} & 1
\end{array}\right|=\left|\begin{array}{ll}
-t^{-2} & 0 \\
2 t^{-3} & 1
\end{array}\right|=-t^{-2}
\end{gathered}
$$

Jadi fungsi Green:

$$
\begin{aligned}
& G(x, t)=\frac{y_{1}(x) V_{1}(t)+y_{2}(x) V_{2}(t)+y_{3}(x) V_{3}(t)}{W\left[y_{1}(t), y_{2}(t), y_{3}(t)\right]} \\
& G(x, t)=\frac{1.3+x^{-1}(-2 t)+x^{2}\left(-t^{-2}\right)}{-6 t^{-2}} \\
& =-\frac{1}{2} t^{2}+\frac{1}{3} t^{3} x^{-1}+\frac{1}{6} x^{2} \\
& f(t)=\frac{1}{t^{3}}+\frac{1}{t^{4}}
\end{aligned}
$$

Dengan memilih $x_{0}=1$, maka solusi khususnya :

$$
y_{p}=\int_{1}^{x}\left(-\frac{1}{2} t^{2}+\frac{1}{3} t^{3} x^{-1}+\frac{1}{6} x^{2}\right) \cdot\left(\frac{1}{t^{3}}+\frac{1}{t^{4}}\right) d t
$$


Jadi solusi umumnya :

$$
\begin{gathered}
y_{p}=\int_{1}^{x}\left(-\frac{1}{2} t^{-1}-\frac{1}{2} t^{-2}+\frac{1}{3} x^{-1}+\frac{1}{3} t^{-1} x^{-1}+\frac{1}{6} x^{2} t^{-3}+\frac{1}{6} x^{2} t^{-4}\right) d t \\
y_{p}=-\frac{1}{2} \ln |t|+\frac{1}{2} t^{-1}+\frac{1}{3} x^{-1} t+\frac{1}{3} x^{-1} \ln |t|-\frac{1}{12} x^{2} t^{-2}-\left.\frac{1}{18} x^{2} t^{-3}\right|_{1} ^{x} \\
y_{p}=-\frac{1}{2} \ln |x|+\frac{1}{2} x^{-1}+\frac{1}{3}+\frac{1}{3} x^{-1} \ln |x|-\frac{1}{12}-\frac{1}{18} x^{-1}-\frac{1}{2}-\frac{1}{3} x^{-1}+\frac{1}{12} x^{2}+\frac{1}{18} x^{2} \\
y_{p}=\frac{1}{9} x^{-1}+\frac{5}{36} x^{2}-\frac{1}{2} \ln |x|+\frac{1}{3} x^{-1} \ln |x|-\frac{1}{4}
\end{gathered}
$$

$$
\begin{gathered}
y=c_{1}+c_{2} x^{-1}+c_{3} x^{2}+\frac{1}{9} x^{-1}+\frac{5}{36} x^{2}-\frac{1}{2} \ln |x|+\frac{1}{3} x^{-1} \ln |x|-\frac{1}{4} \\
y=A+B x^{-1}+C x^{2}-\frac{1}{2} \ln |x|+\frac{1}{3} x^{-1} \ln |x|
\end{gathered}
$$

\section{Simpulan}

Berdasarkan hasil pembahasan maka diperoleh kesimpulan bahwa melalui variasi parameter dapat membangun fungsi Green suatu persamaan diferensial linear non homogen tingkat- $n$. Tujuannya agar dapat menentukan solusi persamaan diferensial untuk fungsi $f$ sembarang.

\section{Daftar Pustaka}

[1]. Brauer, F., Nohel, J.A., 1968, Problems and Solutions in Ordinary Differential Equatuion,W.A. Benjamin, New York, 131-136.

[2]. Boyce, Wiliam E., and DiPrima, Richard C, 2008, Elementary Differential Equations, ninth edition, John Wiley \& Sons, Inc.

[3]. Boyce, Wiliam E., and DiPrima, Richard C, 2012, Elementary Differential Equations and Boundary Value Problems, 10th edition, John Wiley \& Sons, Inc.

[4]. Carrier, G. F., Pearson,C,E., 1991, Ordinary Differential Equation, SIAM, 64-68.

[5]. E. Williamson, Richard., 1997, Introduction to Differential Equations and Dynamical Systems, The Mc Graw-Hill Company. Inc., New York, St. Louis, San Francisco, Tokyo, Toronto. 
